Business and Economics Research Journal
Volume 9 Number 12018
pp. $187-194$
ISSN: $1309-2448$
www.berjournal.com

\title{
Entropi Temelli ROV Yöntemi İle Esnek Üretim Sistemi Seçimi
}

\begin{abstract}
Alptekin Ulutaşa
Öz: Küresel rekabetin artmasıla çoğu üretim işletmesi rekabet avantajını elde etmek için üretim teknolojilerini geliştirmeye yönelmektedir. Esnek üretim sistemleri de bu üretim teknolojilerinden biridir. Esnek üretim sistemleri, çeşitli ürünleri kısa sürede ve belirlenen miktarlarda üretme kapasitesine sahiptir. Ileri teknolojinin yaygınlaşması ile birlikte üretim yapan işletmeler esnek üretim sisteminin sağladığı avantajlardan faydalanabilmek için bu sistemi kullanmaya başlamıştır. Esnek üretim sistemi değerlendirilirken ya da seçilirken kimi birbiriyle çelişen birçok kriter göz önünde bulundurulmaktadır. Bu sebeple, esnek üretim sistemlerinin değerlendirmesinde veya seçiminde çok kriterli karar verme yöntemleri kullanılmaktadır. Bu çalışmada Entropi temelli değer aralığı (range of value (ROV)) yöntemi ile esnek üretim sistemi seçimi yapılacaktır. Entropi yöntemiyle kriterlerin objektif ağırlıkları elde edilecek ve ROV yöntemi ile alternatif sıralaması yapılacaktır. Yöntemin kullanımı ile sekiz farklı alternatif performanslarına göre sıralanmıştır. Türkçe literatürde ROV yönteminin kullanıldığı çalışma bulunmadığından, bu çalışmanın Türkçe literatüre katkı sunacağı düşünülmektedir.
\end{abstract}

Anahtar Sözcükler: Esnek Üretim Sistemleri, Çok Kriterli Karar Verme, ROV yöntemi, Entropi Yöntemi

JEL Sınıflandırması: M11, C44

\section{Flexible Manufacturing System Selection with Entropy based ROV Method}

Abstract: With increasing global competition, most manufacturing businesses turn to increase their production technologies to gain competitive advantage. Flexible manufacturing systems are also one of these production technologies. Flexible manufacturing systems have the capacity to produce a variety of products in a short time and in specified quantities. With the widespread use of advanced technology, manufacturing companies have begun using flexible manufacturing system to take advantage of benefits of this system. When evaluating or selecting of flexible manufacturing systems, many criteria, which are conflicted, should be considered. Hence, multi-criteria decision making methods are used in the evaluation or selection of flexible manufacturing systems. In this study, the flexible manufacturing systems will be selected with the Entropy based range of value (ROV) method. The objective weights of the criteria will be obtained by the Entropy method and alternatives will be ranked by the ROV method. Eight different alternatives are ranked with respect to their performance by using of this method. Since there is no study using ROV in Turkish literature, this study is thought to contribute to Turkish literature.

Keywords: Flexible Manufacturing Systems, Multi-criteria Decision Making, ROV Method, Entropy Method

JEL Classification: M11, C44

${ }^{a}$ Asst. Prof., PhD., Cumhuriyet University, Faculty of Economics and Administrative Sciences, Department of International Trade and Logistics, Sivas, Turkiye, aulutas@cumhuriyet.edu.tr (ORCID ID: 0000-0002-8130-1301) 


\section{Giriş}

Günümüzde artan küresel rekabet nedeniyle, üretim yapan şirketler müşteri ihtiyaçlarını hızlı bir şekilde karşılamak ve küresel pazarda rekabet avantajını kazanmak için bilgisayar kontrollü makinelere, otomatik malzeme taşıma sistemlerine ve esnek üretim sistemleri (EÜS) gibi gelişmiş üretim teknolojilerine intiyaç duymaktadırlar. EÜS, ileri teknolojinin yaygınlaşması ile birlikte birçok üretim firmasının ilgisini çekmiş ve birçok şirket bu sisteme geçiş yapmıştır. Bir EÜS, parçaları ve/veya araçları hareket ettirmek için fiziksel olarak bir nakil ağı ile birbirine bağlanmış CNC takım tezgâhlarından ve/veya robotlardan ve genel bir bilgisayar kontrolünden oluşmaktadır (Karsak, 2008). EÜS'nin "esnek" olarak nitelenmesinin nedeni, çeşitli iş istasyonlarında farklı parça türlerinin aynı anda işlenebilmesi ve parça türlerinin karışımı ile bunların üretim miktarlarının, değişen talep miktarına uygun olarak kolayca ayarlanabilmesidir (Chatterjee ve Chakraborty, 2014). EÜS'nin kullanımının potansiyel faydaları şunlardır; stok seviyelerini azaltır, üretimin tedarik süresini azaltır, kurulum maliyetlerini azaltır, yüksek esneklik ve kalite sağlar (Karsak, 2008).

EÜS'leri, kısa sürede çeşitli ürünleri istenilen miktarda üretme kapasitesine sahip olan sistemlerdir. Bundan dolayı EÜs'ye sahip olmak birçok üretici firma için artık bir gereklilik olmaya başlamıştır. Bunun yanında EÜS'nin kurulumu büyük bir sermaye yatırımı gerektirdiği için en uygun EÜS'nin seçimi kapsamlı analiz ve değerlendirme gerektirmektedir (Chatterjee ve Chakraborty, 2014). Dolayısıyla, EÜs'nin seçiminde sistemden en yüksek faydayı sağlayabilmek için detaylı bir kriter taramasının yapııması ve bu kriterlerin önem sıralamalarının doğru şekilde belirlenmesi gerekmektedir. Bu kriterlerden bir bölümünün birbirleriyle çelişmesi durumunda ölçüm güçleşmekte ve üretim işletmesindeki karar vericilerin tercihleri de hesaba katıldığında bu kriterler arasında bir denge oluşturulması gerekmektedir (Chatterjee ve Chakraborty, 2014). EÜS'nin seçim aşamasına çok sayıda kriter dahil edildiğinden, EÜS Seçiminde literatürde birçok kez çok kriterli karar verme yöntemi kullanılmıştir.

Literatürde EÜS seçimi için kullanılmış çok kriterli karar verme yöntemlerinden bazıları şu şekildedir; analitik hiyerarşi süreci (AHS) (Chan vd., 2000; Bayazit, 2005), uzlaşma derecelendirme yöntemi (Rao, 2009), TOPSIS (Karsak, 2002; Rao, 2008) ve aksiyomatik tasarım yöntemi (Kulak ve Kahraman, 2005). Son yıllarda EÜS değerlendirmesi ve seçimi için yapılan çalışmalardan birisi, EÜS seçimi için birden fazla çok kriterli karar verme yönteminin birlikte kullanıldığı Chatterjee ve Chakraborty (2014)'nin çalışmasıdır. Yazarlar, kriter ağırlıklarını belirlemede Entropi yöntemini kullanmışlardır ve EÜS'lerin sıralanmasında tercih sıralama yöntemleri olarak adlandırdıkları yöntemleri (EVAMIX, COPRAS, EXPROM 2, ORESTE, OCRA ve ARAS) kullanmışlardır. Bir diğer çalışmada Talebanpour ve Javadi (2015), DEMATEL ve basit ağırlıklı toplama (BAT) yöntemlerini kullanarak EÜS kurulumunun yapılıp yapılmaması kararlarını değerlendirmişlerdir. DEMATEL yöntemi kriterler arasındaki ilişki yoğunluğunu bulmak ve kriterlerin ağırlığını hesaplamak için kullanılmıştır. BAT yöntemi ise EÜS kurulumunun yapılıp yapılmaması hakkındaki kararları değerlendirmiştir. Bir başka çalışmada, Ervural vd. (2016) sezgisel (hesitant) bulanık mantık ile çok özellikli grup karar verme yöntemini birleştirerek esnek üretim sistemlerini değerlendirmişlerdir.

Bu çalışmada EÜS seçimi için Entropi Temelli Değer Aralığı (Range of Value) (ROV) yöntemi kullanılacaktır. Bu çalışma, ROV yöntemi ile yapılmış Türkçe bir çalışma olmadığından orijinaldir. Daha önce Entropi ve ROV yöntemleri sadece bir çalışmada bir araya gelmiş ve elma seçimi için kullanılmıştır (Işık ve Adalı, 2017). Yapılan çalışmadan farklı olarak bu çalışmada yöntemin uygulama alanı EÜS seçimi olarak belirlenmiştir. Bu çalışmada örnekte kullanılan karar matrisi bilindiği için objektif ağırlıkların bulunmasında Entropi yöntemi kullanılmıştır. Bir diğer objektif ağırlıkları bulma yöntemi olan CRITIC yöntemi ise daha çok işlem gerektirdiği için bu çalışmada tercih edilmemiştir. PROMETHEE ve ELECTRE gibi çok kriterli karar verme yöntemlerinde alternatif sayısının artması işlem süresini artırmaktadır (Özdağoğlu, 2013). ROV yönteminde alternatif sayısı artsa bile, ikili karşılaştırma yapılmadığı için bu yöntemlere kıyasla işlem süresi çok kısa olmaktadır. Bu yüzden ROV yöntemi bu çalışmada tercih edilmiştir. 


\section{Metodoloji}

Bu çalışmada Entropi temelli ROV yöntemi kullanılmıştır. Entropi yöntemi kriter ağırlıklarını belirlemek amacıyla, ROV yöntemi ise EÜS seçiminde kullanılmıştır.

\subsection{Entropi Yöntemi}

Entropi kavramı, bilginin içinde olan belirsizliği ölçmek için kullanılmaktadır. Bilginin entropisi bir sistemin düzensizlik derecesinin ölçülmesidir ve entropi eldeki verilerle yararlı bilgilerin miktarını ölçebilir (Işık ve Adalı, 2017). Entropi yöntemi bir ağırlıklandırma yöntemi olup, birçok yayında çok kriterli karar verme probleminin çözümünde kullanılmıştr. Örneğin, Shemshadi vd. (2011) tedarikçi seçimi için bulanık VIKOR ve Entropi yöntemini kullanmışlardır. Bir diğer çalışmada, Zhengyuan vd. (2011) bölgesel enerji şebekesi şirketinin operasyon kabiliyetini değerlendirmek için Entropi yöntemi ile bulanık kapsamlı (Fuzzy Comprehensive) metodunu birleştirmişlerdir. Zhang vd. (2011) turizm rekabetçiliğinin değerlendirilmesi için TOPSIS ve bilgi Entropisi kullanmışlardır. Safari vd. (2012) tedarikçi seçimi için PROMETHEE ve Entropi yöntemlerini birleştirmişlerdir. Zhao ve Guo (2014) bulanık Entropi ve bulanık TOPSIS yöntemlerini kullanarak termal güç ekipmanlarının alımı için yeşil tedarikçi seçimi yapmışlardır. Sun vd. (2016) ağ servislerini değerlendirmek için TOPSIS ve Entropi yöntemlerini kullanmışlardır. Basuki (2016) KOBi'ler için sürdürülebilir strateji seçimi için Entropi ve VIKOR yöntemlerini kullanmıştir. Ömürbek vd. (2017) Entropi-Aras ve Entropi-Moosra yöntemlerini kullanarak yaşam kalitesi açısından AB ülkelerini değerlendirmişlerdir. Akçakanat vd. (2017) Entropi ve WASPAS yöntemlerini kullanarak bankacılık sektöründe performans ölçümü yapmışlardır.

Bu yöntem, 6 adımda özetlenebilir (Wang ve Lee, 2009; Li vd., 2011):

Adım 1-1: İlk aşama olarak kriterlerin ve seçeneklerin yer aldığı bir karar matrisi oluşturulur. Eşitlik 1 karar matrisini göstermektedir:

$$
D=\left[y_{i j}\right]_{m \times n}=\left[\begin{array}{cccc}
y_{11} & y_{12} & \cdots & y_{1 n} \\
y_{21} & y_{22} & \cdots & y_{2 n} \\
\vdots & \vdots & \vdots & \vdots \\
y_{m 1} & y_{m 2} & \cdots & y_{m n}
\end{array}\right]
$$

Eşitlik 1 'de yer alan $y_{i j}, i$. alternatifin $j$. kriterde gösterdiği performansı ifade etmektedir. Bu karar matrisinde toplam $n$ adet kriter ve $m$ adet alternatif yer almaktadır.

Adım 1-2: Eşitlik 2 ve 3'teki denklemler yardımıyla karar matrisindeki değerler standartlaştırılır. Eşitlik 2, faydalı kriterler için kullanılırken eşitlik 3 faydasız kriterler için kullanılmaktadır. Eşitliklerde yer alan $r_{i j}$ değerleri, $y_{i j}$ değerinin standartlaşmış halini göstermektedir.

$$
\begin{aligned}
& r_{i j}=\frac{y_{i j}}{\max _{j}\left(y_{i j}\right)} \\
& r_{i j}=\frac{\min _{j}\left(y_{i j}\right)}{y_{i j}}, \min _{j}\left(y_{i j}\right) \neq 0
\end{aligned}
$$

Adım 1-3: Standartlaşmış değerler eşitlik 4 yardımı ile normalize edilir. Eşitlik 4'deki $t_{i j}$ değeri normalize edilmiş değeri göstermektedir.

$$
t_{i j}=\frac{r_{i j}}{\sum_{i=1}^{m} r_{i j}}
$$

Adım 1-4: Normalizasyon işleminin ardından, her bir kriterin entropi değeri eşitlik 5 'in kullanımı ile elde edilir. Aşağıdaki eşitlikte gösterilen $H_{j}$ değeri $j$. kriterin entropisini göstermektedir.

$$
H_{j}=-\frac{\sum_{i=1}^{m} t_{i j} \ln \left(t_{i j}\right)}{\ln (m)}
$$


Adım 1-5: Son adımda her bir kriterin ağırlığı eşitlik 6 yardımıyla elde edilir.

$w_{j}=\frac{1-H_{j}}{\sum_{j=1}^{n}\left(1-H_{j}\right)}$

Yukarıdaki eşitlikte gösterilen $w_{j}$ değeri $j$. kriterin objektif ağırlığını göstermektedir. Entropi yönteminin sonucunda objektif kriter ağırlıkları elde edilir. Bu yöntemin son adımından sonra ROV yöntemine geçilir ve bu yöntem sayesinde bulunan objektif ağırlıklar ROV yöntemine aktarılır.

\subsection{ROV Yöntemi}

ROV (Range of Value) (Değer Aralığı) yöntemi 1993 yılında Yakowitz ve arkadaşları tarafindan geliştirilmiştir (Yakowitz vd., 1993). ROV yönteminin, genellikle karar vericilerin kriterlere ağırlık vermede sıkıntı yaşadıkları durumlarda kullanılmasının çok faydalı olduğu görülmüştür (Madic vd., 2016; Işık ve Adalı, 2017). Örneğimizde karar vericiden herhangi bir veri alınmadığından ROV yöntemi kullanılmıştır. Bu yöntem kolay uygulanabilirliğe sahiptir ve üç adımdan oluşmaktadır; karar matrisinin oluşturulması, normalize edilmesi ve fayda fonksiyonlarının bulunması. Karmaşık bir işlem içermediği ve işlem süresinin kısalığından dolayı birçok çok kriterli karar verme problemine uygulanabilir ve hızlıca sonuca ulaşılabilir. Literatürde ROV yöntemi az sayıda yayında kullanılmıştır. Örneğin, Hajkowicz ve Higgins (2008) su yönetim problemine ağırlıklı toplam, ROV, PROMETHEE II, Evamix ve uzlaşma programlamasından oluşan bir çok kriterli analiz sistemi önermiştir. Athawele ve Chakraborty (2011) ROV yöntemini robot seçiminde kullanmışlardır ve diğer çok kriterli karar verme yöntemleri ile karşılaştırmışlardır. Madić vd. (2015), lazer kesiminin çok amaçlı optimizasyonu için ROV tabanlı bir Taguchi metodolojisi geliştirmişlerdir. Madić vd. (2016) kesme sıvıSı seçiminde ROV yöntemini kullanmışlardır. ROV yöntemi 3 adımda özetlenebilir (Hajkowicz ve Higgins ,2008)

Adım 2-1: Eşitlik 1'de gösterilen $D$ karar matrisi seçeneklerin kriterlerde gösterdikleri performansları göstermektedir. Dolayısıyla, ROV yöntemine bu karar matrisi ile devam edilir.

Adım 2-2: Karar matrisinde yer alan her bir performans değeri eşitlik 7 (faydalı kriterler için) ve 8 (faydasız kriterler için) ile normalize edilir (Zavadskas ve Turskis, 2010).

$$
\begin{aligned}
& \bar{y}_{i j}=\frac{y_{i j}}{\sum_{i=1}^{m} y_{i j}} \\
& \bar{y}_{i j}=\frac{\left(1 / y_{i j}\right)}{\sum_{i=1}^{m}\left(1 / y_{i j}\right)}
\end{aligned}
$$

Adım 2-3: Son adımda her bir alternatifin en iyi ve en kötü fayda fonksiyonları hesaplanır. Bu işlemi gerçekleştirmek için faydalı ve faydasız kriterler için ayrı ayrı fayda fonksiyonları oluşturulur. Faydalı ve faydasız kriterler için fayda fonksiyonları $\left(u_{i}^{+}\right.$ve $\left.u_{i}^{-}\right)$sırasıyla eşitlik 9 ve $10^{\prime}$ da gösterilmiştir.

$$
\begin{aligned}
& \text { Artırma: } u_{i}^{+}=\sum_{j=1}^{n} \bar{y}_{i j} w_{j} \\
& \text { Azaltma: } u_{i}^{-}=\sum_{j=1}^{n} \bar{y}_{i j} w_{j}
\end{aligned}
$$

Eşitlik 9 ve 10'da gösterilen $w_{j}$ Entropi yönteminden elde edilmiştir. Ağırlıklar mutlaka şu iki şart sağlamak zorundadırlar; $\sum_{j=1}^{n} w_{j}=1$ ve $w_{j} \geq 0$.

Eğer $u_{i}^{-}>u_{i^{\prime}}^{+}$ise $i$ alternatifi toplam skora bakılmadan $i^{\prime}$ alternatifinden iyi denilebilir. Eğer bu gerçekleşmiyorsa aşağıdaki formülle orta nokta bulunarak buna göre sıralama yapılır. 
$u_{i}=\frac{u_{i}^{+}+u_{i}^{-}}{2}$

\section{Sayısal Örnek}

Entropi temelli ROV yönteminin geçerliliğini test etmek için Rao ve Parnichkun (2009)'un makalesindeki örnek çözülmüştür. Rao ve Parnichkun (2009) bir EÜS seçilim problemi için kombinasyonel matematik tabanlı yaklaşım önermiştir. Bu problem için sekiz alternatif ve yedi kriter belirlemiştir. Bu yedi kriter şöyledir; işçilik maliyetinde düşüş (IMD), süreç adımları arasında biriken iş miktarındaki azalma yüzdesi (SAiMAY), kurulum maliyetindeki azalma yüzdesi (KMAY), piyasa tepkisindeki artş̧ (PTA), kalitedeki artş̧ (KA), sermaye ve bakım maliyeti (SBM) (bin dolar), kullanılan taban alanı (KTA) (metrekare). Bu yedi kriterden ilk beş kriter faydalı kriter ve son iki kriter ise faydasız kriter olarak belirlenmiştir. Tablo 1 problemin karar matrisini göstermektedir.

Tablo 1. Karar Matrisi

\begin{tabular}{|c|c|c|c|c|c|c|c|}
\hline Kriterler & IMD & SAIMAY & KMAY & PTA & KA & SBM & KTA \\
Alternatif & & & & & & & \\
\hline Alternatif 1 & 30 & 23 & 5 & 0,745 & 0,745 & 1500 & 5000 \\
\hline Alternatif 2 & 18 & 13 & 15 & 0,745 & 0,745 & 1300 & 6000 \\
\hline Alternatif 3 & 15 & 12 & 10 & 0,5 & 0,5 & 950 & 7000 \\
\hline Alternatif 4 & 25 & 20 & 13 & 0,745 & 0,745 & 1200 & 4000 \\
\hline Alternatif 5 & 14 & 18 & 14 & 0,255 & 0,745 & 950 & 3500 \\
\hline Alternatif 6 & 17 & 15 & 9 & 0,745 & 0,5 & 1250 & 5250 \\
\hline Alternatif 7 & 23 & 18 & 20 & 0,5 & 0,745 & 1100 & 3000 \\
\hline Alternatif 8 & 16 & 8 & 14 & 0,255 & 0,5 & 1500 & 3000 \\
\hline
\end{tabular}

Kaynak: Rao ve Parnichkun (2009).

Karar matrisine Entropi yönteminin ilk adımı uygulanarak karar matrisi standartlaştırılır. Tablo 2 standartlaşmış karar matrisini göstermektedir.

Tablo 2. Standartlaşmış Karar Matrisi

\begin{tabular}{|c|c|c|c|c|c|c|c|}
\hline Kriterler & \multirow{2}{*}{ iMD } & SAiMAY & KMAY & PTA & KA & SBM & KTA \\
Alternatif & & & & & & & \\
\hline Alternatif 1 & 1 & 1 & 0,25 & 1 & 1 & 0,633333 & 0,6 \\
\hline Alternatif 2 & 0,6 & 0,565217 & 0,75 & 1 & 1 & 0,730769 & 0,5 \\
\hline Alternatif 3 & 0,5 & 0,521739 & 0,5 & 0,671141 & 0,671141 & 1 & 0,428571 \\
\hline Alternatif 4 & 0,833333 & 0,869565 & 0,65 & 1 & 1 & 0,791667 & 0,75 \\
\hline Alternatif 5 & 0,466667 & 0,782609 & 0,7 & 0,342282 & 1 & 1 & 0,857143 \\
\hline Alternatif 6 & 0,566667 & 0,652174 & 0,45 & 1 & 0,671141 & 0,76 & 0,571429 \\
\hline Alternatif 7 & 0,766667 & 0,782609 & 1 & 0,671141 & 1 & 0,863636 & 1 \\
\hline Alternatif 8 & 0,533333 & 0,347826 & 0,7 & 0,342282 & 0,671141 & 0,633333 & 1 \\
\hline Toplam & 5,266667 & 5,521739 & 5 & 6,026846 & 7,013423 & 6,412739 & 5,707143 \\
\hline
\end{tabular}

Standartlaşmış karar matrisi eşitlik 4 ile normalize edilir. Tablo 3 normalize edilmiş karar matrisini göstermektedir. 
Entropi Temelli ROV Yöntemi Ile Esnek Üretim Sistemi Seçimi

Tablo 3. Normalize Edilmiş Karar Matrisi (Entropi)

\begin{tabular}{|c|c|c|c|c|c|c|c|}
\hline Kriterler & \multirow{2}{*}{ iMD } & SAiMAY & KMAY & PTA & KA & SBM & KTA \\
Alternatif & & & & & & & \\
\hline Alternatif 1 & 0,189873 & 0,181102 & 0,05 & 0,165924 & 0,142584 & 0,098762 & 0,105131 \\
\hline Alternatif 2 & 0,113924 & 0,102362 & 0,15 & 0,165924 & 0,142584 & 0,113956 & 0,08761 \\
\hline Alternatif 3 & 0,094937 & 0,094488 & 0,1 & 0,111359 & 0,095694 & 0,15594 & 0,075094 \\
\hline Alternatif 4 & 0,158228 & 0,15748 & 0,13 & 0,165924 & 0,142584 & 0,123452 & 0,131414 \\
\hline Alternatif 5 & 0,088608 & 0,141732 & 0,14 & 0,056793 & 0,142584 & 0,15594 & 0,150188 \\
\hline Alternatif 6 & 0,107595 & 0,11811 & 0,09 & 0,165924 & 0,095694 & 0,118514 & 0,100125 \\
\hline Alternatif 7 & 0,14557 & 0,141732 & 0,2 & 0,111359 & 0,142584 & 0,134675 & 0,175219 \\
\hline Alternatif 8 & 0,101266 & 0,062992 & 0,14 & 0,056793 & 0,095694 & 0,098762 & 0,175219 \\
\hline
\end{tabular}

Entropi yönteminin son adımı gerçekleştirilerek kriter ağılıklarına ulaşılır. Tablo 4, Entropi yöntemi ile bulunan kriter ağılıklarını göstermektedir.

Tablo 4. Kriter Ağırlıkları

\begin{tabular}{|c|c|c|c|c|c|c|c|}
\hline Kriterler & IMD & SAiMAY & KMAY & PTA & KA & SBM & KTA \\
\hline Ağırlıklar & 0,120653 & 0,14941 & 0,213282 & 0,256091 & 0,060419 & 0,049658 & 0,150487 \\
\hline
\end{tabular}

Kriter ağırlıkları bulunduktan sonra ROV yöntemine geçilir. Eşitlik 7 ve 8 yardımı ile karar matrisi ROV yöntemi için normalize edilir. Tablo 5 normalize edilmiş karar matrisini göstermektedir.

Tablo 5. Normalize Edilmiş Karar Matrisi (ROV)

\begin{tabular}{|c|c|c|c|c|c|c|c|}
\hline Kriterler & \multirow{2}{*}{ iMD } & SAiMAY & KMAY & PTA & KA & SBM & KTA \\
Alternatif & & & & & & & \\
\hline Alternatif 1 & 0,189873 & 0,181102 & 0,05 & 0,165924 & 0,142584 & 0,098762 & 0,105131 \\
\hline Alternatif 2 & 0,113924 & 0,102362 & 0,15 & 0,165924 & 0,142584 & 0,113956 & 0,08761 \\
\hline Alternatif 3 & 0,094937 & 0,094488 & 0,1 & 0,111359 & 0,095694 & 0,15594 & 0,075094 \\
\hline Alternatif 4 & 0,158228 & 0,15748 & 0,13 & 0,165924 & 0,142584 & 0,123452 & 0,131414 \\
\hline Alternatif 5 & 0,088608 & 0,141732 & 0,14 & 0,056793 & 0,142584 & 0,15594 & 0,150188 \\
\hline Alternatif 6 & 0,107595 & 0,11811 & 0,09 & 0,165924 & 0,095694 & 0,118514 & 0,100125 \\
\hline Alternatif 7 & 0,14557 & 0,141732 & 0,2 & 0,111359 & 0,142584 & 0,134675 & 0,175219 \\
\hline Alternatif 8 & 0,101266 & 0,062992 & 0,14 & 0,056793 & 0,095694 & 0,098762 & 0,175219 \\
\hline
\end{tabular}

Tablo 6. Sonuçlar

\begin{tabular}{|c|c|c|c|c|}
\hline Sonuçlar & & & & \\
Alternatif & & $u_{i}^{+}$ & $u_{i}^{-}$ & Siralama \\
\hline Alternatif 1 & 0,111738 & 0,020725 & 0,066232 & 3 \\
\hline Alternatif 2 & 0,112138 & 0,018843 & 0,06549 & 4 \\
\hline Alternatif 3 & 0,0812 & 0,019044 & 0,050122 & 8 \\
\hline Alternatif 4 & 0,121453 & 0,025907 & 0,07368 & 2 \\
\hline Alternatif 5 & 0,084885 & 0,030345 & 0,057615 & 6 \\
\hline Alternatif 6 & 0,098097 & 0,020953 & 0,059525 & 5 \\
\hline Alternatif 7 & 0,118529 & 0,033056 & 0,075792 & 1 \\
\hline Alternatif 8 & 0,071815 & 0,031273 & 0,051544 & 7 \\
\hline
\end{tabular}


Normalize edilmiş karar matrisinden sonra ROV yönteminin son adımına geçilir ve eşitlik 11 yardımıyla EÜS'lerin son skorları belirlenmiş olunur. Tablo 6, EÜS'lerin son skorlarını ve sıralamalarını göstermektedir.

Rao ve Parnichkun (2009) makalesinde kriter ağırlıklarını belirlemek için analitik hiyerarşi sürecini (AHS) kullanmıştır ve kriter ağırlıklarını şu şekilde belirlemiştir; $w_{\mathrm{I} M D}=0,1181, \quad w_{S A \dot{I} M A Y}=0,1181$, $w_{K M A Y}=0,0445, w_{P T A}=0,1181, w_{K A}=0,2861, w_{S B M}=0,2861$ ve $w_{K T A}=0,0445$. Ayrıca, Rao ve Parnichkun (2009) EÜS'lerin sıralamasını 3-4-7-2-5-6-1-8 olarak belirlemiştir. Entropi temelli ROV yönteminin sonuçlarına göre sıralama 3-4-8-2-6-5-1-7 olarak bulundu. Kriter ağırlıklarının çözüme etki edeceğini düşünerek Rao ve Parnichkun (2009)'un makalesinde yer alan AHS kriter ağırlıkları, ROV yöntemine aktarılmıştır ve Entropi yönteminden elde edilen ağırlıklar yerine kullanılmıştır. AHS temelli ROV yönteminin sonuçlarına göre sıralama şu şekilde olmuştur; 3-5-7-1-4-6-2-8. Bu üç yöntemin sonuçları Spearman sıralama korelasyon katsayısı kullanılarak incelenmiştir. Sonuçlara göre AHS temelli ROV yöntemi ile Entropi temelli ROV yöntemi arasındaki korelasyon katsayısı 0,881 olarak, Entropi temelli ROV yöntemi ile Rao ve Parnichkun (2009)'un yöntemi arasındaki korelasyon katsayısı 0,952 ve son olarak AHS temelli ROV yöntemi ile Rao ve Parnichkun (2009)'un yöntemi arasındaki korelasyon katsayısı 0,952 olarak bulunmuştur. Korelasyon katsayılarına bakılarak ROV yönteminin EÜS seçiminde kullanılmasının uygun olduğu görülmüştür.

\section{Sonuçlar}

Esnek üretim sistemleri istenilen çeşit ürünü istenilen miktarda üretebildiği için çok önemli sistemlerdir. Birçok üretici firma EÜs'nin sağladığı avantajlardan yararlanabilmek için esnek üretim sistemi kurmaktadır. Esnek üretim sistemi seçiminde veya değerlendirilmesinde birçok kriter göz önünde bulundurulur. Ayrıca bu kriterler bazen birbiri ile çelişmektedir. Bu nedenle EÜS seçiminde ve değerlendirmesinde çok kriterli karar verme yöntemleri kullanılması uygundur. Bu çalışmada EÜS seçimi için Entropi temelli ROV yöntemi önerilmiştir. Entropi yöntemi EÜS seçiminde kullanılan kriterlerin objektif ağırlıklarını bulmak için kullanılmıştır. ROV yöntemi ise esnek üretim sistemlerinin kriterlerde gösterdikleri performanslarına göre bu sistemleri sıralamıştır. Bu çalışmanın temel amacı ROV yöntemini Türkçe literatüre tanıtmaktır. Daha önce Türkçe literatürde ROV yöntemi ile ilgili bir çalışma bulunamamıştır. Bu yüzden bu çalışma orijinaldir. Ayrıca, ROV yöntemi az işlem içermektedir ve hızlı bir şekilde sağlıklı çözümlere ulaşmaktadır. Bundan dolayı, ROV yöntemi bu problemin çözümünde kullanılmıştır. Gelecek çalışmalarda ROV yöntemi diğer karar verme problemlerinde(depo yeri seçimi, personel seçimi, tedarikçi seçimi vb.) uygulanabilir.

\section{Kaynaklar}

Akçakanat, Ö., Eren, H., Aksoy, E., \& Ömürbek, V. (2017). Bankaclık sektöründe entropi ve waspas yöntemleri ile performans değerlendirmesi. Süleyman Demirel Üniversitesi iktisadi ve Idari Bilimler Fakültesi Dergisi, 22 (2), 285300.

Athawale, V. M., \& Chakraborty, S. (2011). A comparative study on the ranking performance of some multi-criteria decision-making methods for industrial robot selection. International Journal of Industrial Engineering Computations, 2(4), 831-850.

Bayazit, O. (2005). Use of AHP in decision-making for flexible manufacturing systems. Journal of Manufacturing Technology Management, 16(7), 808-819.

Basuki, A. (2016). Sustainable strategies selection in SMEs using MCDM approach. In MATEC Web of Conferences (Vol. 58). EDP Sciences (pp.1-6).

Chan, F. T., Jiang, B., \& Tang, N. K. (2000). The development of intelligent decision support tools to aid the design of flexible manufacturing systems. International Journal of Production Economics, 65(1), 73-84.

Chatterjee, P., \& Chakraborty, S. (2014). Flexible manufacturing system selection using preference ranking methods: A comparative study. International Journal of Industrial Engineering Computations, 5(2), 315-338.

Ervural, B. Ç., Ervural, B., \& Kabak, Ö. (2016). A Group decision making approach for the evaluation of flexible manufacturing systems. IFAC-PapersOnLine, 49(12), 1329-1334. 
Hajkowicz, S., \& Higgins, A. (2008). A comparison of multiple criteria analysis techniques for water resource management. European Journal of Operational Research, 184(1), 255-265.

Işık, A. T., \& Adalı, E. A. (2017). The decision-making approach based on the combination of entropy and ROV methods for the apple selection problem. EJIS European Journal of Interdisciplinary Studies Articles, 8(1), 80-86.

Karsak, E. E. (2002). Distance-based fuzzy MCDM approach for evaluating flexible manufacturing system alternatives. International Journal of Production Research, 40(13), 3167-3181.

Karsak, E. E. (2008). Using data envelopment analysis for evaluating flexible manufacturing systems in the presence of imprecise data. The International Journal of Advanced Manufacturing Technology, 35(9-10), 867-874.

Kulak, O., \& Kahraman, C. (2005). Multi-attribute comparison of advanced manufacturing systems using fuzzy vs. crisp axiomatic design approach. International Journal of Production Economics, 95(3), 415-424.

Li, X., Wang, K., Liu, L., Xin, J., Yang, H., \& Gao, C. (2011). Application of the entropy weight and TOPSIS method in safety evaluation of coal mines. Procedia Engineering, 26, 2085-2091.

Madić, M., Radovanović, M., Coteata, M., Janković, P., \& Petković, D. (2015). Multi-objective optimization of laser cutting using ROV-based taguchi methodology. In Applied Mechanics and Materials (Vol. 809, pp. 405-410). Trans Tech Publications.

Madić, M., Radovanović, M., \& Manić, M. (2016). Application of the ROV method for the selection of cutting fluids. Decision Science Letters, 5(2), 245-254.

Ömürbek, N., Eren, H., \& Dağ, O. (2017). Entropi-Aras ve Entropi-moosra yöntemleri ile yaşam kalitesi açısından $A B$ ülkelerinin değerlendirilmesi. Ömer Halisdemir Üniversitesi Iktisadi ve Idari Bilimler Fakültesi Dergisi, 10(2):2948.

Özdağoğlu, A. (2013). İmalat işletmeleri için eksantrik pres alternatiflerinin COPRAS yöntemi ile karşılaştırılması. Gümüşhane Üniversitesi Sosyal Bilimler Enstitüsü Dergisi, 4(8), 1-22.

Rao, R. V. (2008). Evaluating flexible manufacturing systems using a combined multiple attribute decision making method. International Journal of Production Research, 46(7), 1975-1989.

Rao, R. V. (2009). Flexible manufacturing system selection using an improved compromise ranking method. International Journal of Industrial and Systems Engineering , 4(2), 198-215.

Rao, R. V., \& Parnichkun, M. (2009). Flexible manufacturing system selection using a combinatorial mathematics-based decision-making method. International Journal of Production Research, 47(24), 6981-6998.

Safari, H., Fagheyi, M. S., Ahangari, S. S., \& Fathi, M. R. (2012). Applying Promethee method based on entropy weight for supplier selection. Business Management and Strategy, 3(1), 97-106.

Shemshadi, A., Shirazi, H., Toreihi, M., \& Tarokh, M. J. (2011). A fuzzy VIKOR method for supplier selection based on entropy measure for objective weighting. Expert Systems with Applications, 38(10), 12160-12167.

Sun, R., Zhang, B., \& Liu, T. (2016, May). Ranking web service for high quality by applying improved Entropy-TOPSIS method. In Software Engineering, Artificial Intelligence, Networking and Parallel/Distributed Computing (SNPD), 2016 17th IEEE/ACIS International Conference on (pp. 249-254). IEEE.

Talebanpour, R., \& Javadi, M. (2015). Decision-making for flexible manufacturing systems using DEMATEL and SAW. Decision Science Letters, 4(3), 363-372.

Wang, T. C., \& Lee, H. D. (2009). Developing a fuzzy TOPSIS approach based on subjective weights and objective weights. Expert Systems with Applications, 36(5), 8980-8985.

Yakowitz, D. S., Lane, L. J., \& Szidarovszky, F. (1993). Multi-attribute decision making: Dominance with respect to an importance order of the attributes. Applied Mathematics and Computation, 54(2-3), 167-181.

Zavadskas, E. K., \& Turskis, Z. (2010). A new additive ratio assessment (ARAS) method in multicriteria decisionmaking. Technological and Economic Development of Economy, 16(2), 159-172.

Zhang, H., Gu, C. L., Gu, L. W., \& Zhang, Y. (2011). The evaluation of tourism destination competitiveness by TOPSIS \& information entropy-A case in the Yangtze River Delta of China. Tourism Management, 32(2), 443-451.

Zhao, H., \& Guo, S. (2014). Selecting green supplier of thermal power equipment by using a hybrid MCDM method for sustainability. Sustainability, 6(1), 217-235.

Zhengyuan, J., Chunmei, W., Zhiwei, H., \& Gang, Z. (2011). Evaluation research of regional power grid companies' operation capacity based on entropy weight fuzzy comprehensive model. Procedia Engineering, 15, 4626-4630. 